\title{
Comparative analysis of some reliability characteristics between two dissimilar redundant systems with replacement at common cause failure
}

\author{
Ibrahim Yusuf ${ }^{1 *}$, Bashir Yusuf ${ }^{2}$ \\ ${ }^{1}$ Department of Mathematics, Bayero University, Kano, Nigeria \\ ${ }^{2}$ Department of Mathematics, Federal University, Dutse, Nigeria \\ *Corresponding author E-mail: Ibrahimyusuffagge@gmail.com
}

Copyright $\odot 2014$ Ibrahim Yusuf, Bashir Yusuf. This is an open access article distributed under the Creative Commons Attribution License, which permits unrestricted use, distribution, and reproduction in any medium, provided the original work is properly cited.

\begin{abstract}
In this paper, probabilistic models for two dissimilar redundant systems with replacement at each common cause failure have been developed to analyze and compare some reliability characteristics. Two configurations are studied under the assumption that each system is replaced at the occurrence of common cause failure. Configuration I is a 3-out-of-4 cold standby system, while configuration II is 3-out-of-5 cold standby system. Explicit expressions for mean time to system failure (MTSF), steady-state availability, busy period and profit function for the three models are analyzed using Kolmogorov's forward equations method. Comparisons are performed for specific values of system parameters. Furthermore, we compare these reliability characteristics for the two configurations and find that configuration II is more reliable and efficient than configuration I.
\end{abstract}

Keywords: Redundancy, replacement, Common cause failure.

\section{Introduction}

Redundancy is a technique used to improve system reliability and availability. Reliability optimizations play a key role in engineering design and have been effectively applied to enhance performance [4], [17]. One of the forms of redundancy is the k-out-of-n system which has wide application in industrial setting. Moreover, the k-out-of-n system works if and only if at least $\mathrm{k}$ of the $\mathrm{n}$ components work. Due to their importance in industries and design, the k-out-of$\mathrm{n}$ systems have received attention from different researchers (see, for instance, [1], [9], [16] and the references therein). The concept of common cause failure and its impact on reliability measure of system effectiveness has been introduced by several authors such as [2], who studied common cause failure analysis of a non-identical unit parallel repairable system with arbitrary distributed repair times. Furthermore, [15] studied cost analysis of a system involving common cause failures and preventive maintenance. [3] Has analyzed the reliability of redundant system with common cause failure. [8] Performed computational comparisons of confidence intervals for the steady-state availability of a repairable system. [5] Performed comparative analysis between two unit cold standby and warm standby outdoor electric power systems in changing weather. [11] Performed comparative analysis of availability between three systems with general repair times, reboot delay and switching failures. [12] Performed comparative analysis of availability between two systems with warm standby units and different imperfect coverage. [6] Performed comparative analysis of some reliability characteristics between redundant systems requiring supporting units for their operation. Many researchers have studied reliability problem of different systems (see, for instance [7], [14]). The problem considered in this paper is different from the work of [2], [3], and [15]. In this paper, we studied 3-out-of-4 and 3-out-of-5 cold standby systems involving replacement at each common cause failure and derived their corresponding mathematical model using Kolmogorov's forward equation method. The contributions of this paper are threefold. First is to develop the explicit expressions for, and for configuration. The second is to determine the impact of failure rate, repair rate, common cause failure and replacement rate on, and for configuration. The third is to rank the two configurations for the, and based on assumed numerical values given to the system parameters. The organization of the paper is as follows. In Section 2, we give the notations, assumptions and states of the systems. System models formulation are given in Section 3 . The results 
of our numerical simulations and discussions of the results are presented in Section 4. Finally, we make a concluding remark in Section 5.

\section{Notations, assumptions and states of the systems}

\subsection{Notations}

$\beta_{i} / \alpha_{i}:$ Failure/repair rate of unit $i, i=1,2,3,4$

$\lambda_{1} / \mu_{1}$ : Common cause failure/replacement rate for unit $i, i=2,3,4$

$\lambda_{2} / \mu_{2}$ : Common cause failure/ replacement rate for unit $i, i=1,3,4$

$\lambda_{3} / \mu_{3}$ : Common cause failure/ replacement rate for unit $i, i=1,2,4$

$M T S F_{J}$ : Mean time to system failure for configuration I and II, $J=1,2$

$A_{V J}$ : Steady-state availability for configuration I and II, $J=1,2$

$B_{r J}$ : Busy period due to repair for configuration I and II, $J=1,2$

$B_{R J}$ : Busy period due to replacement for configuration I and II, $J=1,2$

$B_{J}$ : Busy period for configuration I and II, $J=1,2$

$P F_{J}$ : Profit function for configuration I and II, $J=1,2$

$O_{N} / O_{R} / O_{W} / O_{G} / O_{C}:$ Unit is normal/under repair/waiting for repair/idle/under common cause failure $S_{i}, i=0,1,2,3,4:$ States of the system

\subsection{Assumptions}

1) Configuration I and II consists of three operative and one and two cold standby units respectively.

2) At common cause failure, the affected units are replaced with new ones.

3) Switching from standby to operative unit is perfect and instantaneous.

4) Each system is attended by one repairman.

5) Repair is perfect.

6) The common cause failure affects only the units in operation and the units are replaced instantaneously.

\subsection{States of the systems}

Configuration I: 3-out-of-4 system

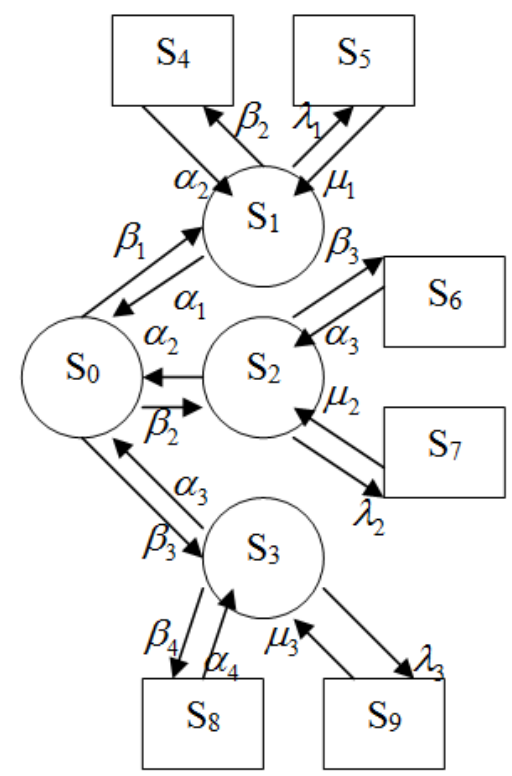

Fig. 1: Transition diagram of Configuration

Up states 
$S_{0}\left(O_{1 N}, O_{2 N}, O_{3 N}, O_{4 S}\right), S_{1}\left(O_{1 R}, O_{2 N}, O_{3 N}, O_{4 N}\right), S_{2}\left(O_{1 N}, O_{2 R}, O_{3 N}, O_{4 N}\right), S_{3}\left(O_{1 N}, O_{2 N}, O_{3 R}, O_{4 N}\right)$

Down states:

$S_{4}\left(O_{1 R}, O_{2 w}, O_{3 G}, O_{4 G}\right) \quad, \quad S_{5}\left(O_{1 R}, O_{2 C}, O_{3 C}, O_{4 C}\right) \quad, \quad S_{6}\left(O_{1 G}, O_{2 R}, O_{3 W}, O_{4 G}\right) \quad, \quad S_{7}\left(O_{1 C}, O_{2 R}, O_{3 C}, O_{4 C}\right) \quad$, $S_{8}\left(O_{1 G}, O_{2 G}, O_{3 R}, O_{4 W}\right), S_{9}\left(O_{1 C}, O_{2 C}, O_{3 R}, O_{4 C}\right)$

Configuration II: 3 -out-of-5 system

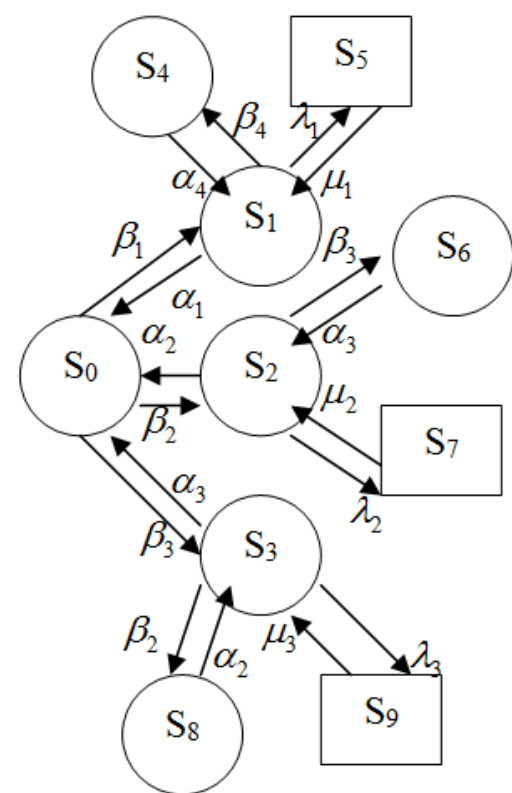

Fig. 2: Transition diagram of Configuration II

Up states

$S_{0}\left(O_{1 N}, O_{2 N}, O_{3 N}, O_{4 S}, O_{5 S}\right), S_{1}\left(O_{1 R}, O_{2 N}, O_{3 N}, O_{4 N}, O_{5 S}\right), S_{2}\left(O_{1 N}, O_{2 R}, O_{3 N}, O_{4 N}, O_{5 S}\right), S_{3}\left(O_{1 N}, O_{2 N}, O_{3 R}, O_{4 N}, O_{5 S}\right)$,

$S_{4}\left(O_{1 R}, O_{2 N}, O_{3 N}, O_{4 W}, O_{5 N}\right), S_{6}\left(O_{1 N}, O_{2 R}, O_{3 W}, O_{4 N}, O_{5 N}\right)$

$S_{8}\left(O_{1 N}, O_{2 W}, O_{3 R}, O_{4 N}, O_{5 N}\right)$

Down states

$S_{5}\left(O_{1 R}, O_{2 C}, O_{3 C}, O_{4 C}, O_{5 G}\right), S_{7}\left(O_{1 C}, O_{2 R}, O_{3 C}, O_{4 C}, O_{5 G}\right), S_{9}\left(O_{1 C}, O_{2 C}, O_{3 R}, O_{4 C}, O_{5 G}\right)$

\section{Model formulation}

Let $P_{i}(t)$ to be the probability that the systems at time $t \geq 0$ are in the states $S_{i}, i=0,1,2, \ldots, 9$. Also let $P_{n}(t)$, $n=I, I I$ be the probability row vector at time $t$, we have the following initial conditions for configuration I and II respectively:

$P_{n}(0)=\left[P_{0}(0), P_{1}(0), P_{2}(0), P_{3}(0), P_{4}(0), P_{5}(0), P_{6}(0), P_{7}(0), P_{8}(0), P_{9}(0)\right]$

$=[1,0,0,0,0,0,0,0,0,0]$

We obtain the following differential equations from Fig. 1 and Fig. 2 for configuration I and II respectively.

$\frac{d p_{0}(t)}{d t}=-\left(\beta_{1}+\beta_{2}+\beta_{3}\right) p_{0}(t)+\alpha_{1} p_{1}(t)+\alpha_{2} p_{2}(t)+\alpha_{3} p_{3}(t)$

$\frac{d p_{1}(t)}{d t}=-\left(\alpha_{1}+\lambda_{1}+\beta_{2}\right) p_{1}(t)+\beta_{1} p_{0}(t)+\alpha_{2} p_{4}(t)+\mu_{1} p_{5}(t)$

$\frac{d p_{2}(t)}{d t}=-\left(\alpha_{2}+\lambda_{2}+\beta_{3}\right) p_{2}(t)+\beta_{2} p_{0}(t)+\alpha_{3} p_{6}(t)+\mu_{2} p_{7}(t)$

$\frac{d p_{3}(t)}{d t}=-\left(\alpha_{3}+\lambda_{3}+\beta_{4}\right) p_{3}(t)+\beta_{3} p_{0}(t)+\alpha_{4} p_{4}(t)+\mu_{3} p_{9}(t)$

$\frac{d p_{4}(t)}{d t}=-\alpha_{2} p_{4}(t)+\beta_{2} p_{1}(t)$

$\frac{d p_{5}(t)}{d t}=-\mu_{1} p_{5}(t)+\lambda_{1} p_{1}(t)$ 


$$
\begin{aligned}
& \frac{d p_{6}(t)}{d t}=-\alpha_{3} p_{6}(t)+\beta_{3} p_{2}(t) \\
& \frac{d p_{7}(t)}{d t}=-\mu_{2} p_{7}(t)+\lambda_{2} p_{2}(t) \\
& \frac{d p_{8}(t)}{d t}=-\alpha_{4} p_{8}(t)+\beta_{4} p_{3}(t) \\
& \frac{d p_{9}(t)}{d t}=-\mu_{3} p_{9}(t)+\lambda_{3} p_{3}(t)
\end{aligned}
$$

The differential equations in (1) above can be transformed into matrix as: $\dot{P}_{1}=T_{1} P_{1}$

Where

$T_{1}=\left[\begin{array}{cccccccccc}-\left(\beta_{1}+\beta_{2}+\beta_{3}\right) & \alpha_{1} & \alpha_{2} & \alpha_{3} & 0 & 0 & 0 & 0 & 0 & 0 \\ \beta_{1} & -\left(\alpha_{1}+\lambda_{1}+\beta_{2}\right) & 0 & 0 & \alpha_{2} & \mu_{1} & 0 & 0 & 0 & 0 \\ \beta_{2} & 0 & -\left(\alpha_{2}+\lambda_{2}+\beta_{3}\right) & 0 & 0 & 0 & \alpha_{3} & \mu_{2} & 0 & 0 \\ \beta_{3} & 0 & 0 & -\left(\alpha_{3}+\lambda_{3}+\beta_{4}\right) & 0 & 0 & 0 & 0 & \alpha_{4} & \mu_{3} \\ 0 & \beta_{2} & 0 & 0 & -\alpha_{2} & 0 & 0 & 0 & 0 & 0 \\ 0 & \lambda_{1} & 0 & 0 & 0 & -\mu_{1} & 0 & 0 & 0 & 0 \\ 0 & 0 & \beta_{3} & 0 & 0 & 0 & -\alpha_{3} & 0 & 0 & 0 \\ 0 & 0 & \lambda_{2} & 0 & 0 & 0 & 0 & -\mu_{2} & 0 & 0 \\ 0 & 0 & 0 & \beta_{4} & 0 & 0 & 0 & 0 & -\alpha_{4} & 0 \\ 0 & 0 & 0 & \lambda_{3} & 0 & 0 & 0 & 0 & 0 & -\mu_{3}\end{array}\right]$

$\frac{d p_{0}(t)}{d t}=-\left(\beta_{1}+\beta_{2}+\beta_{3}\right) p_{0}(t)+\alpha_{1} p_{1}(t)+\alpha_{2} p_{2}(t)+\alpha_{3} p_{3}(t)$

$\frac{d p_{1}(t)}{d t}=-\left(\alpha_{1}+\lambda_{1}+\beta_{4}\right) p_{1}(t)+\beta_{1} p_{0}(t)+\alpha_{4} p_{4}(t)+\mu_{1} p_{5}(t)$

$\frac{d p_{2}(t)}{d t}=-\left(\alpha_{2}+\lambda_{2}+\beta_{3}\right) p_{2}(t)+\beta_{2} p_{0}(t)+\alpha_{3} p_{6}(t)+\mu_{2} p_{7}(t)$

$\frac{d p_{3}(t)}{d t}=-\left(\alpha_{3}+\lambda_{3}+\beta_{2}\right) p_{3}(t)+\beta_{3} p_{0}(t)+\alpha_{2} p_{8}(t)+\mu_{3} p_{9}(t)$

$\frac{d p_{4}(t)}{d t}=-\alpha_{4} p_{4}(t)+\beta_{4} p_{1}(t)$

$\frac{d p_{5}(t)}{d t}=-\mu_{1} p_{5}(t)+\lambda_{1} p_{1}(t)$

$\frac{d p_{6}(t)}{d t}=-\alpha_{3} p_{6}(t)+\beta_{3} p_{2}(t)$

$\frac{d p_{7}(t)}{d t}=-\mu_{2} p_{7}(t)+\lambda_{2} p_{2}(t)$

$\frac{d p_{8}(t)}{d t}=-\alpha_{2} p_{8}(t)+\beta_{2} p_{3}(t)$

$\frac{d p_{9}(t)}{d t}=-\mu_{3} p_{9}(t)+\lambda_{3} p_{3}(t)$

The differential equations in (3) above can be transformed into matrix as: $\dot{P}_{2}=T_{2} P_{2}$

Where 


$$
T_{2}=\left[\begin{array}{cccccccccc}
-\left(\beta_{1}+\beta_{2}+\beta_{3}\right) & \alpha_{1} & \alpha_{2} & \alpha_{3} & 0 & 0 & 0 & 0 & 0 & 0 \\
\beta_{1} & -\left(\alpha_{1}+\lambda_{1}+\beta_{4}\right) & 0 & 0 & \alpha_{4} & \mu_{1} & 0 & 0 & 0 & 0 \\
\beta_{2} & 0 & -\left(\alpha_{2}+\lambda_{2}+\beta_{3}\right) & 0 & 0 & 0 & \alpha_{3} & \mu_{2} & 0 & 0 \\
\beta_{3} & 0 & 0 & -\left(\alpha_{3}+\lambda_{3}+\beta_{2}\right) & 0 & 0 & 0 & 0 & \alpha_{2} & \mu_{3} \\
0 & \beta_{4} & 0 & 0 & -\alpha_{4} & 0 & 0 & 0 & 0 & 0 \\
0 & \lambda_{1} & 0 & 0 & 0 & -\mu_{1} & 0 & 0 & 0 & 0 \\
0 & 0 & \beta_{3} & 0 & 0 & 0 & -\alpha_{3} & 0 & 0 & 0 \\
0 & 0 & \lambda_{2} & 0 & 0 & 0 & 0 & -\mu_{2} & 0 & 0 \\
0 & 0 & 0 & \beta_{2} & 0 & 0 & 0 & 0 & -\alpha_{2} & 0 \\
0 & 0 & 0 & \lambda_{3} & 0 & 0 & 0 & 0 & 0 & -\mu_{3}
\end{array}\right]
$$

\subsection{Mean time to system failure analysis}

It is difficult to evaluate the transient solutions, hence we follow the Refs. [4,5,11], the procedure to develop the explicit expressions for $M T S F_{1}$ and $M T S F_{2}$ for configuration I and II is to delete the fifth row and column, sixth row and column, seventh row and column, eighth row and column, ninth row and column, and tenth row and column of matrices $T_{1}$ and $T_{2}$ for absorbing states which yield new matrices, say $Q_{1}$ and $Q_{2}$. The expected time to reach an absorbing state is obtained from

$E\left[T_{P(0) \rightarrow P(\text { absorting })}\right]=M T S F_{1}=P_{I}(0)\left(-Q_{1}^{-1}\right)\left[\begin{array}{l}1 \\ 1 \\ 1 \\ 1\end{array}\right]=\frac{N_{1}}{D_{1}}$

For configuration I and

$E\left[T_{P(0) \rightarrow P \text { (absorobing })}\right]=M T S F_{2}=P_{I I}(0)\left(-Q_{2}^{-1}\right)\left[\begin{array}{l}1 \\ 1 \\ 1 \\ 1 \\ 1 \\ 1 \\ 1\end{array}\right]=\frac{N_{2}}{D_{2}}$

For configuration II. Thus,

$$
\begin{aligned}
& \operatorname{MTSF}_{1}=\frac{N_{1}}{D_{1}} \\
& N_{1}=\left(\alpha_{1}+\beta_{2}+\lambda_{1}\right)\left(\alpha_{2}+\beta_{3}+\lambda_{2}\right)\left(\alpha_{3}+\beta_{4}+\lambda_{3}\right)+\beta_{1}\left(\alpha_{2}+\beta_{3}+\lambda_{2}\right)\left(\alpha_{3}+\beta_{4}+\lambda_{3}\right)+\beta_{2}\left(\alpha_{1}+\beta_{2}+\lambda_{1}\right)\left(\alpha_{3}+\beta_{4}+\lambda_{3}\right) \\
& +\beta_{1}\left(\alpha_{1}+\beta_{2}+\lambda_{1}\right)\left(\alpha_{2}+\beta_{3}+\lambda_{2}\right) \\
& D_{1}=\beta_{3}^{2} \beta_{4} \lambda_{1}+\beta_{3}^{2} \lambda_{1} \lambda_{3}+\alpha_{3} \beta_{1} \beta_{2} \lambda_{2}+\beta_{1} \beta_{2} \beta_{4} \lambda_{2}+\beta_{1} \beta_{2} \lambda_{2} \lambda_{3}+\alpha_{3} \beta_{1} \lambda_{1} \lambda_{2}+\beta_{1} \beta_{4} \lambda_{1} \lambda_{2}+\beta_{1} \lambda_{1} \lambda_{2} \lambda_{3}+\alpha_{2} \beta_{2} \beta_{3} \beta_{4}+ \\
& \alpha_{2} \beta_{2} \beta_{3} \lambda_{3}+\alpha_{1} \alpha_{3} \beta_{2} \lambda_{2}+\alpha_{1} \beta_{2} \beta_{4} \lambda_{2}+\alpha_{1} \beta_{2} \lambda_{2} \lambda_{3}+\alpha_{1} \beta_{3} \beta_{4} \lambda_{2}+\alpha_{1} \beta_{3} \lambda_{2} \lambda_{3}+\alpha_{2} \alpha_{3} \beta_{1} \beta_{2}+\alpha_{2} \beta_{1} \beta_{2} \beta_{4}+\alpha_{2} \beta_{1} \beta_{2} \lambda_{3}+ \\
& \alpha_{3} \beta_{1} \beta_{2} \beta_{3}+\beta_{1} \beta_{2} \beta_{3} \beta_{4}+\beta_{1} \beta_{2} \beta_{3} \lambda_{3}+\alpha_{2} \alpha_{3} \beta_{1} \lambda_{1}+\alpha_{2} \beta_{1} \beta_{4} \lambda_{1}+\alpha_{2} \beta_{1} \lambda_{1} \lambda_{3}+\alpha_{3} \beta_{1} \beta_{3} \lambda_{1}+\alpha_{3} \beta_{2}^{2} \beta_{3}+\beta_{2}^{2} \beta_{3} \beta_{4}+\beta_{2}^{2} \beta_{3} \lambda_{3}+ \\
& \alpha_{3} \beta_{2}^{2} \lambda_{2}+\beta_{2}^{2} \beta_{4} \lambda_{2}+\beta_{2}^{2} \lambda_{2} \lambda_{3}+\alpha_{1} \beta_{3}^{2} \beta_{4}+\alpha_{1} \beta_{3}^{2} \lambda_{3}+\beta_{2} \beta_{3}^{2} \beta_{4}+\beta_{2} \beta_{3}^{2} \lambda_{3}+\beta_{1} \beta_{3} \beta_{4} \lambda_{1}+\beta_{1} \beta_{3} \lambda_{1} \lambda_{3}+\alpha_{1} \alpha_{3} \beta_{2} \beta_{3}+ \\
& \alpha_{1} \beta_{2} \beta_{3} \beta_{4}+\alpha_{1} \beta_{2} \beta_{3} \lambda_{3}+\alpha_{3} \beta_{2} \beta_{3} \lambda_{1}+\beta_{2} \beta_{3} \beta_{4} \lambda_{1}+\beta_{2} \beta_{3} \lambda_{1} \lambda_{3}+\alpha_{3} \beta_{2} \lambda_{1} \lambda_{2}+\beta_{2} \beta_{4} \lambda_{1} \lambda_{2}+\beta_{2} \lambda_{1} \lambda_{2} \lambda_{3}+\alpha_{1} \alpha_{2} \beta_{3} \beta_{4}+ \\
& \alpha_{1} \alpha_{2} \beta_{3} \lambda_{3}+\beta_{2} \beta_{3} \beta_{4} \lambda_{2}+\beta_{2} \beta_{3} \lambda_{2} \lambda_{3}+\alpha_{2} \beta_{3} \beta_{4} \lambda_{1}+\alpha_{2} \beta_{3} \lambda_{1} \lambda_{2}+\beta_{3} \beta_{4} \lambda_{1} \lambda_{2}+\beta_{3} \lambda_{1} \lambda_{2} \lambda_{3} \\
& \operatorname{MTSF}_{2}=\frac{N_{2}}{D_{2}} \\
& N_{2}=\alpha_{2} \alpha_{3} \alpha_{4}\left(\alpha_{2} \alpha_{3} \lambda_{1}+\alpha_{2} \lambda_{1} \lambda_{3}+\alpha_{3} \lambda_{1} \lambda_{2}+\lambda_{1} \lambda_{2} \lambda_{3}+\alpha_{1} \alpha_{2} \alpha_{3}+\alpha_{1} \alpha_{2} \lambda_{3}+\alpha_{1} \alpha_{3} \lambda_{2}+\alpha_{1} \lambda_{2} \lambda_{3}\right)+\alpha_{2} \alpha_{3} \alpha_{4} \beta_{1}\left(\alpha_{2} \alpha_{3}+\right. \\
& \left.\alpha_{2} \lambda_{3}+\alpha_{3} \lambda_{2}+\lambda_{2} \lambda_{3}\right)+\alpha_{2} \alpha_{3} \alpha_{4} \beta_{2}\left(\alpha_{3} \lambda_{1}+\lambda_{1} \lambda_{3}+\alpha_{1} \alpha_{3}+\alpha_{1} \lambda_{3}\right)+\alpha_{2} \alpha_{3} \alpha_{4} \beta_{3}\left(\alpha_{2} \lambda_{1}+\lambda_{1} \lambda_{2}+\alpha_{1} \alpha_{2}+\alpha_{1} \lambda_{2}\right)+ \\
& \alpha_{2} \alpha_{3} \beta_{1} \beta_{4}\left(\alpha_{2} \alpha_{3}+\alpha_{2} \lambda_{3}+\alpha_{3} \lambda_{2}+\lambda_{2} \lambda_{3}\right)+\alpha_{2} \alpha_{4} \beta_{2} \beta_{3}\left(\alpha_{3} \lambda_{1}+\lambda_{1} \lambda_{3}+\alpha_{1} \alpha_{3}+\alpha_{1} \lambda_{3}\right)+\alpha_{3} \alpha_{4} \beta_{2} \beta_{3}\left(\alpha_{2} \lambda_{1}+\lambda_{1} \lambda_{2}+\alpha_{1} \alpha_{2}+\alpha_{1} \lambda_{2}\right)
\end{aligned}
$$


$D_{2}=\alpha_{2} \alpha_{3} \alpha_{4}\left(\alpha_{2} \alpha_{3} \beta_{1} \lambda_{1}+\alpha_{2} \beta_{1} \lambda_{1} \lambda_{3}+\alpha_{2} \beta_{3} \lambda_{1} \lambda_{3}+\alpha_{3} \beta_{2} \lambda_{1} \lambda_{2}+\alpha_{3} \beta_{1} \lambda_{1} \lambda_{2}+\beta_{2} \lambda_{1} \lambda_{2} \lambda_{3}+\beta_{3} \lambda_{1} \lambda_{2} \lambda_{3}+\beta_{1} \lambda_{1} \lambda_{2} \lambda_{3}+\right.$ $\left.\alpha_{1} \alpha_{2} \beta_{3} \lambda_{3}+\alpha_{1} \alpha_{3} \beta_{2} \lambda_{2}+\alpha_{1} \beta_{2} \lambda_{2} \lambda_{3}+\alpha_{1} \beta_{3} \lambda_{2} \lambda_{3}\right)$

Where

$Q_{1}=\left[\begin{array}{cccc}-\left(\beta_{1}+\beta_{2}+\beta_{3}\right) & \beta_{1} & \beta_{2} & \beta_{3} \\ \alpha_{1} & -\left(\alpha_{1}+\lambda_{1}+\beta_{2}\right) & 0 & 0 \\ \alpha_{2} & 0 & -\left(\alpha_{2}+\lambda_{2}+\beta_{3}\right) & 0 \\ \alpha_{3} & 0 & 0 & -\left(\alpha_{3}+\lambda_{3}+\beta_{4}\right)\end{array}\right]$

And

$Q_{2}=\left[\begin{array}{ccccccc}-\left(\beta_{1}+\beta_{2}+\beta_{3}\right) & \beta_{1} & \beta_{2} & \beta_{3} & 0 & 0 & 0 \\ \alpha_{1} & -\left(\alpha_{1}+\lambda_{1}+\beta_{4}\right) & 0 & 0 & \beta_{4} & 0 & 0 \\ \alpha_{2} & 0 & -\left(\alpha_{2}+\lambda_{2}+\beta_{3}\right) & 0 & 0 & \beta_{3} & 0 \\ \alpha_{3} & 0 & 0 & -\left(\alpha_{3}+\lambda_{3}+\beta_{2}\right) & 0 & 0 & \beta_{2} \\ 0 & \alpha_{4} & 0 & 0 & -\alpha_{4} & 0 & 0 \\ 0 & 0 & \alpha_{3} & 0 & 0 & -\alpha_{3} & 0 \\ 0 & 0 & 0 & \alpha_{2} & 0 & 0 & -\alpha_{2}\end{array}\right]$

\subsection{Availability Analysis}

For the analysis of availability case, we use the same initial conditions as in section 3 for configuration I.

The differential equations in (1) above can be expressed in matrix form as

$\left[\begin{array}{c}\dot{p}_{0} \\ \dot{p_{1}} \\ \dot{p_{2}} \\ \dot{p_{3}} \\ \dot{p_{4}} \\ \dot{p_{5}} \\ \dot{p}_{6} \\ \dot{p}_{7} \\ \dot{p}_{8} \\ \dot{p}_{9}\end{array}\right]=\left[\begin{array}{cccccccccc}-\left(\beta_{1}+\beta_{2}+\beta_{3}\right) & \alpha_{1} & \alpha_{2} & \alpha_{3} & 0 & 0 & 0 & 0 & 0 & 0 \\ \beta_{1} & -\left(\alpha_{1}+\lambda_{1}+\beta_{2}\right) & 0 & 0 & \alpha_{2} & \mu_{1} & 0 & 0 & 0 & 0 \\ \beta_{2} & 0 & -\left(\alpha_{2}+\lambda_{2}+\beta_{3}\right) & 0 & 0 & 0 & \alpha_{3} & \mu_{2} & 0 & 0 \\ \beta_{3} & 0 & 0 & -\left(\alpha_{3}+\lambda_{3}+\beta_{4}\right) & 0 & 0 & 0 & 0 & \alpha_{4} & \mu_{3} \\ 0 & \beta_{2} & 0 & 0 & -\alpha_{2} & 0 & 0 & 0 & 0 & 0 \\ 0 & \lambda_{1} & 0 & 0 & 0 & -\mu_{1} & 0 & 0 & 0 & 0 \\ 0 & 0 & \beta_{3} & 0 & 0 & 0 & -\alpha_{3} & 0 & 0 & 0 \\ 0 & 0 & \lambda_{2} & 0 & 0 & 0 & 0 & -\mu_{2} & 0 & 0 \\ p_{1}(t) \\ p_{2}(t) \\ p_{3}(t) \\ p_{4}(t) \\ p_{5}(t) \\ p_{6}(t) \\ p_{7}(t) \\ p_{8}(t) \\ p_{9}(t)\end{array}\right]$

Let $V_{1}$ be the time to failure of the system for configuration $I$. Following [11], the steady-state availability is given by

$A_{V 1}(\infty)=1-\left(P_{4}(\infty)+P_{5}(\infty)+P_{6}(\infty)+P_{7}(\infty)+P_{8}(\infty)+P_{9}(\infty)\right)$

In steady state, the derivatives of state probabilities become zero, thus (2) becomes

$T_{1} P_{1}=0$

Which is in matrix form as? 


$\left[\begin{array}{cccccccccc}-\left(\beta_{1}+\beta_{2}+\beta_{3}\right) & \alpha_{1} & \alpha_{2} & \alpha_{3} & 0 & 0 & 0 & 0 & 0 & 0 \\ \beta_{1} & -\left(\alpha_{1}+\lambda_{1}+\beta_{2}\right) & 0 & 0 & \alpha_{2} & \mu_{1} & 0 & 0 & 0 & 0 \\ \beta_{2} & 0 & -\left(\alpha_{2}+\lambda_{2}+\beta_{3}\right) & 0 & 0 & 0 & \alpha_{3} & \mu_{2} & 0 & 0 \\ \beta_{3} & 0 & 0 & -\left(\alpha_{3}+\lambda_{3}+\beta_{4}\right) & 0 & 0 & 0 & 0 & \alpha_{4} & \mu_{3} \\ 0 & \beta_{2} & 0 & 0 & -\alpha_{2} & 0 & 0 & 0 & 0 & 0 \\ 0 & \lambda_{1} & 0 & 0 & 0 & -\mu_{1} & 0 & 0 & 0 & 0 \\ 0 & 0 & \beta_{3} & 0 & 0 & 0 & -\alpha_{3} & 0 & 0 & 0 \\ 0 & 0 & \lambda_{2} & 0 & 0 & 0 & 0 & -\mu_{2} & 0 & 0 \\ 0 & 0 & 0 & \beta_{4} & 0 & 0 & 0 & 0 & -\alpha_{4} & 0 \\ 0 & 0 & 0 & \lambda_{3} & 0 & 0 & 0 & 0 & 0 & -\mu_{3}\end{array}\right]\left[\begin{array}{c}p_{0}(t) \\ p_{1}(t) \\ p_{2}(t) \\ p_{3}(t) \\ p_{4}(t) \\ p_{5}(t) \\ p_{6}(t) \\ p_{7}(t) \\ p_{8}(t) \\ p_{9}(t)\end{array}\right]=\left[\begin{array}{l}0 \\ 0 \\ 0 \\ 0 \\ 0 \\ 0 \\ 0 \\ 0 \\ 0 \\ 0\end{array}\right]$

Using the following normalizing condition

$\sum_{k=0}^{9} P_{K}(\infty)=1$

We substitute (9) in the last row of (8) to yield

$\left[\begin{array}{cccccccccc}-\left(\beta_{1}+\beta_{2}+\beta_{3}\right) & \alpha_{1} & \alpha_{2} & \alpha_{3} & 0 & 0 & 0 & 0 & 0 & 0 \\ \beta_{1} & -\left(\alpha_{1}+\lambda_{1}+\beta_{2}\right) & 0 & 0 & \alpha_{2} & \mu_{1} & 0 & 0 & 0 & 0 \\ \beta_{2} & 0 & -\left(\alpha_{2}+\lambda_{2}+\beta_{3}\right) & 0 & 0 & 0 & \alpha_{3} & \mu_{2} & 0 & 0 \\ \beta_{3} & 0 & 0 & -\left(\alpha_{3}+\lambda_{3}+\beta_{4}\right) & 0 & 0 & 0 & 0 & \alpha_{4} & \mu_{3} \\ 0 & \beta_{2} & 0 & 0 & -\alpha_{2} & 0 & 0 & 0 & 0 & 0 \\ 0 & \lambda_{1} & 0 & 0 & 0 & -\mu_{1} & 0 & 0 & 0 & 0 \\ 0 & 0 & \beta_{3} & 0 & 0 & 0 & -\alpha_{3} & 0 & 0 & 0 \\ 0 & 0 & \lambda_{2} & 0 & 0 & 0 & 0 & -\mu_{2} & 0 & 0 \\ 0 & 0 & 0 & \beta_{4} & 0 & 0 & 0 & 0 & -\alpha_{4} & 0 \\ 1 & 1 & 1 & 1 & 1 & 1 & 1 & 1 & 1 & 1\end{array}\right]\left[\begin{array}{c}p_{0}(t) \\ p_{1}(t) \\ p_{2}(t) \\ p_{3}(t) \\ p_{4}(t) \\ p_{5}(t) \\ p_{6}(t) \\ p_{7}(t) \\ p_{8}(t) \\ p_{9}(t)\end{array}\right]=\left[\begin{array}{c}0 \\ 0 \\ 0 \\ 0 \\ 0 \\ 0 \\ 0 \\ 0 \\ 0 \\ 0\end{array}\right]$

Solving this resulting matrix to obtain the steady-state probabilities $P_{4}(\infty), P_{5}(\infty), P_{6}(\infty), P_{7}(\infty), P_{8}(\infty)$ and $P_{9}(\infty)$ in the availability case. The explicit expression for the $A_{V 1}(\infty)$ in (7) is given by

$A_{V 1}(\infty)=\frac{N_{3}}{D_{3}}$

Where

$N_{3}=\alpha_{1} \alpha_{2} \alpha_{3} \alpha_{4} \mu_{1} \mu_{2} \mu_{3}+\alpha_{2} \alpha_{3} \alpha_{4} \beta_{1} \mu_{1} \mu_{2} \mu_{3}+\alpha_{1} \alpha_{3} \alpha_{4} \beta_{2} \mu_{1} \mu_{2} \mu_{3}+\alpha_{1} \alpha_{2} \alpha_{3} \beta_{3} \mu_{1} \mu_{2} \mu_{3}$

$D_{3}=\alpha_{1} \alpha_{3} \alpha_{4} \beta_{2} \mu_{1} \mu_{3} \lambda_{2}+\alpha_{1} \alpha_{2} \alpha_{3} \alpha_{4} \mu_{1} \mu_{2} \mu_{3}+\alpha_{1} \alpha_{2} \beta_{3} \beta_{4} \mu_{1} \mu_{2} \mu_{3}+\alpha_{1} \alpha_{2} \alpha_{4} \beta_{3} \mu_{1} \mu_{2} \lambda_{3}+\alpha_{1} \alpha_{2} \alpha_{4} \beta_{3} \mu_{1} \mu_{2} \mu_{3}+$ $\alpha_{1} \alpha_{3} \alpha_{4} \beta_{2} \mu_{1} \mu_{2} \mu_{3}+\alpha_{1} \alpha_{4} \beta_{2} \beta_{3} \mu_{1} \mu_{2} \mu_{3}+\alpha_{2} \alpha_{3} \alpha_{4} \beta_{1} \mu_{1} \mu_{2} \mu_{3}+\alpha_{3} \alpha_{4} \beta_{1} \beta_{2} \mu_{1} \mu_{2} \mu_{3}+\alpha_{2} \alpha_{3} \alpha_{4} \beta_{1} \mu_{2} \mu_{3} \lambda_{1}$

For the analysis of availability case, we use the same initial condition as in section 3 for configuration II The differential equations in (3) above can be expressed as 


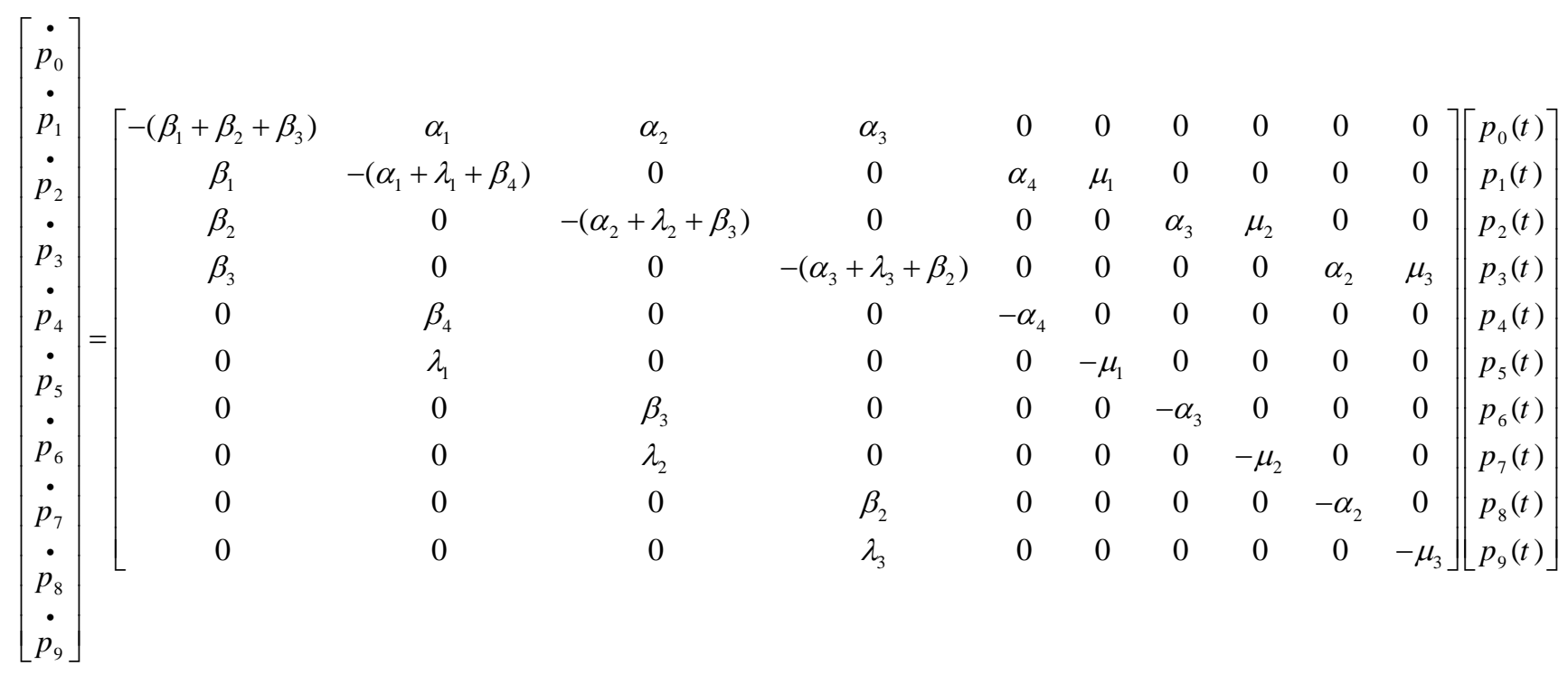

Let $V_{2}$ be the time to failure of the system for configuration $I I$. Following [11], the steady-state availability is given by $A_{V 2}(\infty)=1-\left(P_{5}(\infty)+P_{7}(\infty)+P_{9}(\infty)\right)$

In steady state, the derivatives of state probabilities become zero, thus (4) becomes $T_{2} P_{2}=0$

Which is in matrix form as?

$\left[\begin{array}{cccccccccc}-\left(\beta_{1}+\beta_{2}+\beta_{3}\right) & \alpha_{1} & \alpha_{2} & \alpha_{3} & 0 & 0 & 0 & 0 & 0 & 0 \\ \beta_{1} & -\left(\alpha_{1}+\lambda_{1}+\beta_{4}\right) & 0 & 0 & \alpha_{4} & \mu_{1} & 0 & 0 & 0 & 0 \\ \beta_{2} & 0 & -\left(\alpha_{2}+\lambda_{2}+\beta_{3}\right) & 0 & 0 & 0 & \alpha_{3} & \mu_{2} & 0 & 0 \\ \beta_{3} & 0 & 0 & -\left(\alpha_{3}+\lambda_{3}+\beta_{2}\right) & 0 & 0 & 0 & 0 & \alpha_{2} & \mu_{3} \\ 0 & \beta_{4} & 0 & 0 & -\alpha_{4} & 0 & 0 & 0 & 0 & 0 \\ 0 & \lambda_{1} & 0 & 0 & 0 & -\mu_{1} & 0 & 0 & 0 & 0 \\ 0 & 0 & \beta_{3} & 0 & 0 & 0 & -\alpha_{3} & 0 & 0 & 0 \\ 0 & 0 & \lambda_{2} & 0 & 0 & 0 & 0 & -\mu_{2} & 0 & 0 \\ 0 & 0 & 0 & \beta_{2} & 0 & 0 & 0 & 0 & -\alpha_{2} & 0 \\ 0 & 0 & 0 & \lambda_{3} & 0 & 0 & 0 & 0 & 0 & -\mu_{3}\end{array}\right]\left[\begin{array}{c}p_{0}(t) \\ p_{1}(t) \\ p_{2}(t) \\ p_{3}(t) \\ p_{4}(t) \\ p_{5}(t) \\ p_{6}(t) \\ p_{7}(t) \\ p_{8}(t) \\ p_{9}(t)\end{array}\right]=\left[\begin{array}{l}0 \\ 0 \\ 0 \\ 0 \\ 0 \\ 0 \\ 0 \\ 0 \\ 0 \\ 0\end{array}\right]$

We substitute (9) in the last row of (12) to yield which is in matrix form as

$\left[\begin{array}{cccccccccc}-\left(\beta_{1}+\beta_{2}+\beta_{3}\right) & \alpha_{1} & \alpha_{2} & \alpha_{3} & 0 & 0 & 0 & 0 & 0 & 0 \\ \beta_{1} & -\left(\alpha_{1}+\lambda_{1}+\beta_{4}\right) & 0 & 0 & \alpha_{4} & \mu_{1} & 0 & 0 & 0 & 0 \\ \beta_{2} & 0 & -\left(\alpha_{2}+\lambda_{2}+\beta_{3}\right) & 0 & 0 & 0 & \alpha_{3} & \mu_{2} & 0 & 0 \\ \beta_{3} & 0 & 0 & -\left(\alpha_{3}+\lambda_{3}+\beta_{2}\right) & 0 & 0 & 0 & 0 & \alpha_{2} & \mu_{3} \\ 0 & \beta_{4} & 0 & 0 & -\alpha_{4} & 0 & 0 & 0 & 0 & 0 \\ 0 & \lambda_{1} & 0 & 0 & 0 & -\mu_{1} & 0 & 0 & 0 & 0 \\ 0 & 0 & \beta_{3} & 0 & 0 & 0 & -\alpha_{3} & 0 & 0 & 0 \\ 0 & 0 & \lambda_{2} & 0 & 0 & 0 & 0 & -\mu_{2} & 0 & 0 \\ 0 & 0 & 0 & \beta_{2} & 0 & 0 & 0 & 0 & -\alpha_{2} & 0 \\ 1 & 1 & 1 & 1 & 1 & 1 & 1 & 1 & 1 & 1\end{array}\right]\left[\begin{array}{c}p_{0}(t) \\ p_{1}(t) \\ p_{2}(t) \\ p_{3}(t) \\ p_{4}(t) \\ p_{5}(t) \\ p_{6}(t) \\ p_{7}(t) \\ p_{8}(t) \\ p_{9}(t)\end{array}\right]=\left[\begin{array}{l}0 \\ 0 \\ 0 \\ 0 \\ 0 \\ 0 \\ 0 \\ 0 \\ 0 \\ 1\end{array}\right]$

Solving this resulting matrix to obtain the steady-state probabilities $P_{5}(\infty), P_{7}(\infty)$ and $P_{9}(\infty)$ in the availability case.

The explicit expression for the $A_{V 2}(\infty)$ ins (11) is given by $A_{V 2}(\infty)=\frac{N_{4}}{D_{4}}$ 
$N_{4}=\alpha_{1} \alpha_{2} \alpha_{3} \alpha_{4} \mu_{1} \mu_{2} \mu_{3}+\alpha_{2} \alpha_{3} \alpha_{4} \beta_{1} \mu_{1} \mu_{2} \mu_{3}+\alpha_{1} \alpha_{3} \alpha_{4} \beta_{2} \mu_{1} \mu_{2} \mu_{3}+\alpha_{1} \alpha_{2} \alpha_{4} \beta_{3} \mu_{1} \mu_{2} \mu_{3}+\alpha_{2} \alpha_{3} \beta_{1} \beta_{4} \mu_{1} \mu_{2} \mu_{3}+2 \alpha_{1} \alpha_{4} \beta_{2} \beta_{3} \mu_{1} \mu_{2} \mu_{3}$

$D_{4}=\alpha_{2} \alpha_{3} \beta_{1} \beta_{4} \mu_{1} \mu_{2} \mu_{3}+\alpha_{1} \alpha_{2} \alpha_{3} \alpha_{4} \mu_{1} \mu_{2} \mu_{3}+\alpha_{2} \alpha_{2} \alpha_{4} \beta_{1} \mu_{1} \mu_{2} \mu_{3}+\alpha_{1} \alpha_{2} \alpha_{4} \beta_{3} \mu_{1} \mu_{2} \mu_{3}+\alpha_{1} \alpha_{2} \alpha_{4} \beta_{3} \mu_{1} \mu_{2} \mu_{3}+$ $\alpha_{1} \alpha_{3} \alpha_{4} \beta_{2} \mu_{1} \mu_{2} \mu_{3}+\alpha_{1} \alpha_{3} \alpha_{4} \beta_{2} \mu_{1} \mu_{3} \lambda_{2}+2 \alpha_{1} \alpha_{4} \beta_{2} \beta_{3} \mu_{1} \mu_{2} \mu_{3}+\alpha_{2} \alpha_{3} \alpha_{4} \beta_{1} \mu_{2} \mu_{3} \lambda_{1}$

\subsection{Busy period analysis}

Using the same initial condition as for the reliability case $P_{n}(0)=\left[P_{0}(0), P_{1}(0), P_{2}(0), P_{3}(0), P_{4}(0), P_{5}(0), P_{6}(0), P_{7}(0), P_{8}(0), P_{9}(0)\right]$

$=[1,0,0,0,0,0,0,0,0,0]$

And equations (1), (8), and (9) for configuration $I$ to yield $\left[\begin{array}{cccccccccc}-\left(\beta_{1}+\beta_{2}+\beta_{3}\right) & \alpha_{1} & \alpha_{2} & \alpha_{3} & 0 & 0 & 0 & 0 & 0 & 0 \\ \beta_{1} & -\left(\alpha_{1}+\lambda_{1}+\beta_{2}\right) & 0 & 0 & \alpha_{2} & \mu_{1} & 0 & 0 & 0 & 0 \\ \beta_{2} & 0 & -\left(\alpha_{2}+\lambda_{2}+\beta_{3}\right) & 0 & 0 & 0 & \alpha_{3} & \mu_{2} & 0 & 0 \\ \beta_{3} & 0 & 0 & -\left(\alpha_{3}+\lambda_{3}+\beta_{4}\right) & 0 & 0 & 0 & 0 & \alpha_{4} & \mu_{3} \\ 0 & \beta_{2} & 0 & 0 & -\alpha_{2} & 0 & 0 & 0 & 0 & 0 \\ 0 & \lambda_{1} & 0 & 0 & 0 & -\mu_{1} & 0 & 0 & 0 & 0 \\ 0 & 0 & \beta_{3} & 0 & 0 & 0 & -\alpha_{3} & 0 & 0 & 0 \\ 0 & 0 & \lambda_{2} & 0 & 0 & 0 & 0 & -\mu_{2} & 0 & 0 \\ 0 & 0 & 0 & \beta_{4} & 0 & 0 & 0 & 0 & -\alpha_{4} & 0 \\ 1 & 1 & 1 & 1 & 1 & 1 & 1 & 1 & 1 & 1\end{array}\right]\left[\begin{array}{c}p_{0}(t) \\ p_{1}(t) \\ p_{2}(t) \\ p_{3}(t) \\ p_{4}(t) \\ p_{5}(t) \\ p_{6}(t) \\ p_{7}(t) \\ p_{8}(t) \\ p_{9}(t)\end{array}\right]=\left[\begin{array}{l}0 \\ 0 \\ 0 \\ 0 \\ 0 \\ 0 \\ 0 \\ 0 \\ 0 \\ 1\end{array}\right]$ Solving this resulting matrix to obtain the steady-state probabilities $P_{1}(\infty), P_{2}(\infty), P_{3}(\infty), P_{4}(\infty), P_{5}(\infty), P_{6}(\infty), P_{7}(\infty), P_{8}(\infty)$ and $P_{9}(\infty)$

The busy period due to repairs for configuration $I$ am given by

$B_{r 1}(\infty)=P_{1}(\infty)+P_{2}(\infty)+P_{3}(\infty)+P_{4}(\infty)+P_{6}(\infty)+P_{8}(\infty)$

$B_{r 1}(\infty)=\frac{N_{5}}{D_{3}}$

$N_{5}=\alpha_{2} \alpha_{3} \alpha_{4} \beta_{1} \mu_{1} \mu_{2} \mu_{3}+\alpha_{1} \alpha_{3} \alpha_{4} \beta_{2} \mu_{1} \mu_{2} \mu_{3}+\alpha_{1} \alpha_{2} \alpha_{4} \beta_{3} \mu_{1} \mu_{2} \mu_{3}+\alpha_{3} \alpha_{4} \beta_{1} \beta_{2} \mu_{1} \mu_{2} \mu_{3}+\alpha_{1} \alpha_{4} \beta_{2} \beta_{3} \mu_{1} \mu_{2} \mu_{3}+\alpha_{1} \alpha_{2} \beta_{3} \beta_{4} \mu_{1} \mu_{2} \mu_{3}$ $D_{3}=\alpha_{1} \alpha_{3} \alpha_{4} \beta_{2} \mu_{1} \mu_{3} \lambda_{2}+\alpha_{1} \alpha_{2} \alpha_{3} \alpha_{4} \mu_{1} \mu_{2} \mu_{3}+\alpha_{1} \alpha_{2} \beta_{3} \beta_{4} \mu_{1} \mu_{2} \mu_{3}+\alpha_{1} \alpha_{2} \alpha_{4} \beta_{3} \mu_{1} \mu_{2} \lambda_{3}+\alpha_{1} \alpha_{2} \alpha_{4} \beta_{3} \mu_{1} \mu_{2} \mu_{3}+$ $\alpha_{1} \alpha_{3} \alpha_{4} \beta_{2} \mu_{1} \mu_{2} \mu_{3}+\alpha_{1} \alpha_{4} \beta_{2} \beta_{3} \mu_{1} \mu_{2} \mu_{3}+\alpha_{2} \alpha_{3} \alpha_{4} \beta_{1} \mu_{1} \mu_{2} \mu_{3}+\alpha_{3} \alpha_{4} \beta_{1} \beta_{2} \mu_{1} \mu_{2} \mu_{3}+\alpha_{2} \alpha_{3} \alpha_{4} \beta_{1} \mu_{2} \mu_{3} \lambda_{1}$ The busy period due to replacement for configuration $I$ is given by

$B_{R 1}(\infty)=P_{5}(\infty)+P_{7}(\infty)+P_{9}(\infty)$

$B_{R 1}(\infty)=\frac{N_{6}}{D_{3}}$

$N_{6}=\alpha_{2} \alpha_{3} \alpha_{4} \beta_{1} \mu_{2} \mu_{3} \lambda_{1}+\alpha_{1} \alpha_{3} \alpha_{4} \beta_{2} \mu_{1} \mu_{3} \lambda_{2}+\alpha_{1} \alpha_{2} \alpha_{4} \beta_{3} \mu_{1} \mu_{2} \lambda_{3}$

Busy period for configuration $I$ am given by

$B_{1}(\infty)=B_{r 1}(\infty)+B_{R 1}(\infty)$

Using the same initial condition as for the reliability case

$P_{n}(0)=\left[P_{0}(0), P_{1}(0), P_{2}(0), P_{3}(0), P_{4}(0), P_{5}(0), P_{6}(0), P_{7}(0), P_{8}(0), P_{9}(0)\right]$

$=[1,0,0,0,0,0,0,0,0,0]$

And equations (3), (9), and (12) for configuration II to yield 


$\left[\begin{array}{cccccccccc}-\left(\beta_{1}+\beta_{2}+\beta_{3}\right) & \alpha_{1} & \alpha_{2} & \alpha_{3} & 0 & 0 & 0 & 0 & 0 & 0 \\ \beta_{1} & -\left(\alpha_{1}+\lambda_{1}+\beta_{4}\right) & 0 & 0 & \alpha_{4} & \mu_{1} & 0 & 0 & 0 & 0 \\ \beta_{2} & 0 & -\left(\alpha_{2}+\lambda_{2}+\beta_{3}\right) & 0 & 0 & 0 & \alpha_{3} & \mu_{2} & 0 & 0 \\ \beta_{3} & 0 & 0 & -\left(\alpha_{3}+\lambda_{3}+\beta_{2}\right) & 0 & 0 & 0 & 0 & \alpha_{2} & \mu_{3} \\ 0 & \beta_{4} & 0 & 0 & -\alpha_{4} & 0 & 0 & 0 & 0 & 0 \\ 0 & \lambda_{1} & 0 & 0 & 0 & -\mu_{1} & 0 & 0 & 0 & 0 \\ 0 & 0 & \beta_{3} & 0 & 0 & 0 & -\alpha_{3} & 0 & 0 & 0 \\ 0 & 0 & \lambda_{2} & 0 & 0 & 0 & 0 & -\mu_{2} & 0 & 0 \\ 0 & 0 & 0 & \beta_{2} & 0 & 0 & 0 & 0 & -\alpha_{2} & 0 \\ 1 & 1 & 1 & 1 & 1 & 1 & 1 & 1 & 1 & 1\end{array}\right]\left[\begin{array}{c}p_{0}(t) \\ p_{1}(t) \\ p_{2}(t) \\ p_{3}(t) \\ p_{4}(t) \\ p_{5}(t) \\ p_{6}(t) \\ p_{7}(t) \\ p_{8}(t) \\ p_{9}(t)\end{array}\right]=\left[\begin{array}{l}0 \\ 0 \\ 0 \\ 0 \\ 0 \\ 0 \\ 0 \\ 0 \\ 0 \\ 1\end{array}\right]$

Solving this resulting matrix to obtain the steady-state probabilities $P_{1}(\infty), P_{2}(\infty), P_{3}(\infty), P_{4}(\infty), P_{5}(\infty), P_{6}(\infty), P_{7}(\infty), P_{8}(\infty)$ and $P_{9}(\infty)$

The busy period due to repair for configuration II is given by

$B_{r 2}(\infty)=P_{1}(\infty)+P_{2}(\infty)+P_{3}(\infty)+P_{4}(\infty)+P_{6}(\infty)+P_{8}(\infty)$

$B_{r 2}(\infty)=\frac{N_{7}}{D_{4}}$

$N_{7}=\alpha_{2} \alpha_{3} \alpha_{4} \beta_{1} \mu_{1} \mu_{2} \mu_{3}+\alpha_{1} \alpha_{3} \alpha_{4} \beta_{2} \mu_{1} \mu_{2} \mu_{3}+\alpha_{1} \alpha_{2} \alpha_{4} \beta_{3} \mu_{1} \mu_{2} \mu_{3}+\alpha_{2} \alpha_{3} \beta_{1} \beta_{4} \mu_{1} \mu_{2} \mu_{3}+2 \alpha_{1} \alpha_{4} \beta_{2} \beta_{3} \mu_{1} \mu_{2} \mu_{3}$

$D_{4}=\alpha_{2} \alpha_{3} \beta_{1} \beta_{4} \mu_{1} \mu_{2} \mu_{3}+\alpha_{1} \alpha_{2} \alpha_{3} \alpha_{4} \mu_{1} \mu_{2} \mu_{3}+\alpha_{2} \alpha_{2} \alpha_{4} \beta_{1} \mu_{1} \mu_{2} \mu_{3}+\alpha_{1} \alpha_{2} \alpha_{4} \beta_{3} \mu_{1} \mu_{2} \mu_{3}+\alpha_{1} \alpha_{2} \alpha_{4} \beta_{3} \mu_{1} \mu_{2} \mu_{3}+$

$\alpha_{1} \alpha_{3} \alpha_{4} \beta_{2} \mu_{1} \mu_{2} \mu_{3}+\alpha_{1} \alpha_{3} \alpha_{4} \beta_{2} \mu_{1} \mu_{3} \lambda_{2}+2 \alpha_{1} \alpha_{4} \beta_{2} \beta_{3} \mu_{1} \mu_{2} \mu_{3}+\alpha_{2} \alpha_{3} \alpha_{4} \beta_{1} \mu_{2} \mu_{3} \lambda_{1}$

The busy period due to replacement for configuration $I I$ is given by

$B_{R 2}(\infty)=P_{5}(\infty)+P_{7}(\infty)+P_{9}(\infty)$

$B_{R 2}(\infty)=\frac{N_{8}}{D_{4}}$

$N_{8}=\alpha_{2} \alpha_{3} \alpha_{4} \beta_{1} \mu_{2} \mu_{3} \lambda_{1}+\alpha_{1} \alpha_{3} \alpha_{4} \beta_{2} \mu_{1} \mu_{3} \lambda_{2}+\alpha_{1} \alpha_{2} \alpha_{4} \beta_{3} \mu_{1} \mu_{2} \lambda_{3}$

Busy period for configuration II is given by

$B_{2}(\infty)=B_{r 2}(\infty)+B_{R 2}(\infty)$

\subsection{Profit analysis}

The units are subjected to corrective maintenance at failure and replacement at common cause failure as can be observed in states 1, 2, 3,4,6,8 and 5, 7, 8 respectively. From Fig. 1 and 2 the repairman performed corrective maintenance action to the units at failure in states $1,2,3,4,6,8$ and performed replacement to failed units due to common cause failure in states 5, 7 and 8 in both configuration I and II. Let $C_{0}$ and $C_{1}$ be the revenue generated when the system is in working state and no income when in failed state, cost of each repair and replacement respectively. Following [4], [5], the expected total profit per unit time incurred to the system in the steady-state is

Profit=total revenue generated - cost incurred by the repair man due to repair and replacement.

$P F_{1}=C_{0} A_{v 1}(\infty)-C_{1} B_{1}(\infty)$

$P F_{2}=C_{0} A_{v 2}(\infty)-C_{1} B_{2}(\infty)$

\section{Results and discussions}

In this section, we numerically obtained and compare the results for mean time to system failure, system availability and profit function for all the developed models. For each model the following set of parameters values are fixed throughout the simulations for consistency for the two cases with the corresponding results tabulated in each case:

Case I: $\beta_{2}=0.2, \beta_{3}=0.05, \beta_{4}=0.01, \lambda_{2}=0.4, \lambda_{3}=0.5, \alpha_{2}=0.6, \alpha_{3}=0.5, \alpha_{4}=0.9, \mu_{1}=0.6, \mu_{2}=0.6, \mu_{3}=0.5$ for Figures $3-5$.

Case II: $\beta_{2}=0.2, \beta_{3}=0.05, \beta_{4}=0.01, \lambda_{2}=0.4, \lambda_{3}=0.5, \alpha_{2}=0.6, \alpha_{3}=0.5, \alpha_{4}=0.9, \mu_{2}=0.6, \mu_{3}=0.5, C_{0}=1000$, $C_{1}=100$ for Figures $6-13$ 


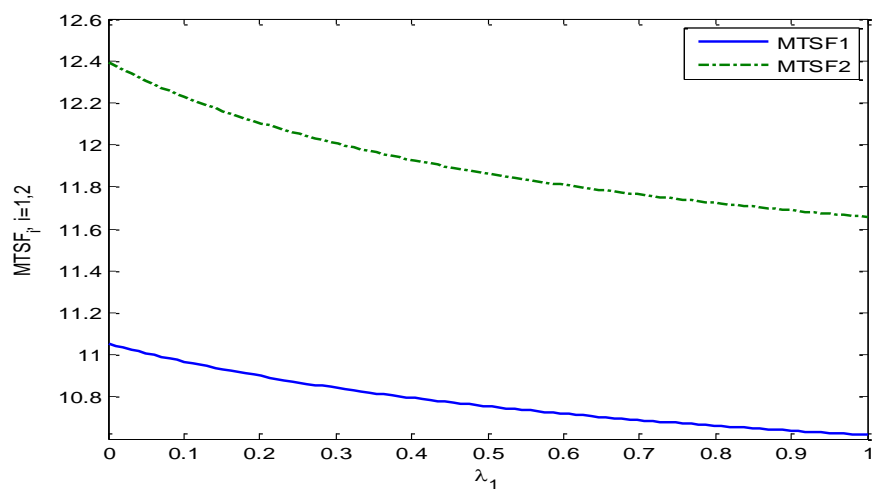

Fig. 3: $M T S F_{i}$ versus common cause failure $\lambda_{1}$

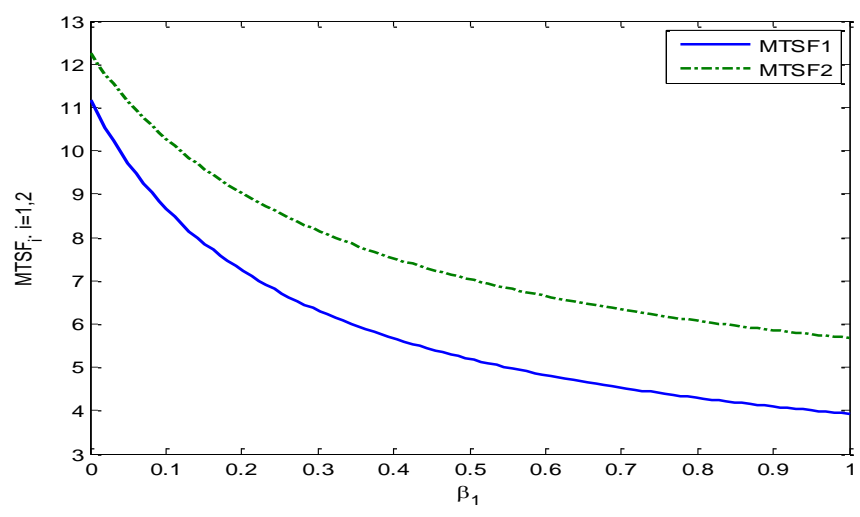

Fig. 4: $M T S F_{i}$ versus failure rate $\beta_{1}$

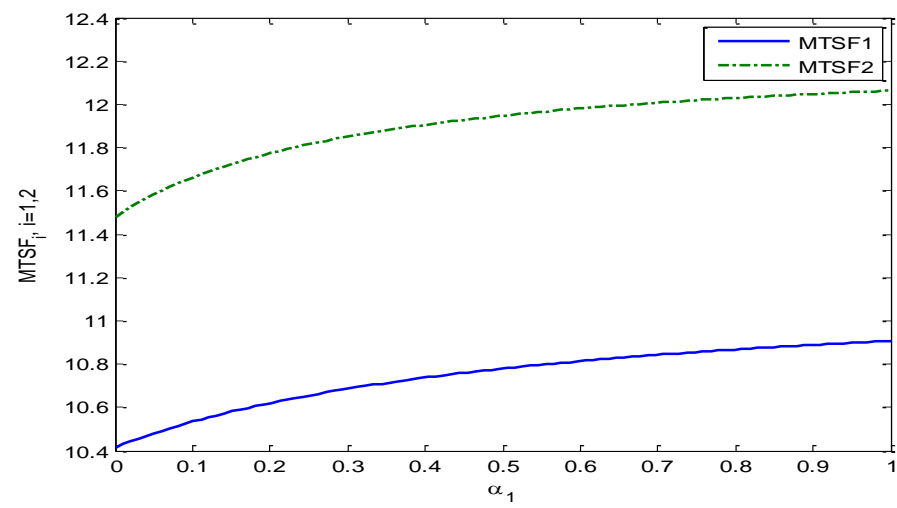

Fig. 5: $\operatorname{MTSF}_{i}$ versus repair rate $\alpha_{1}$

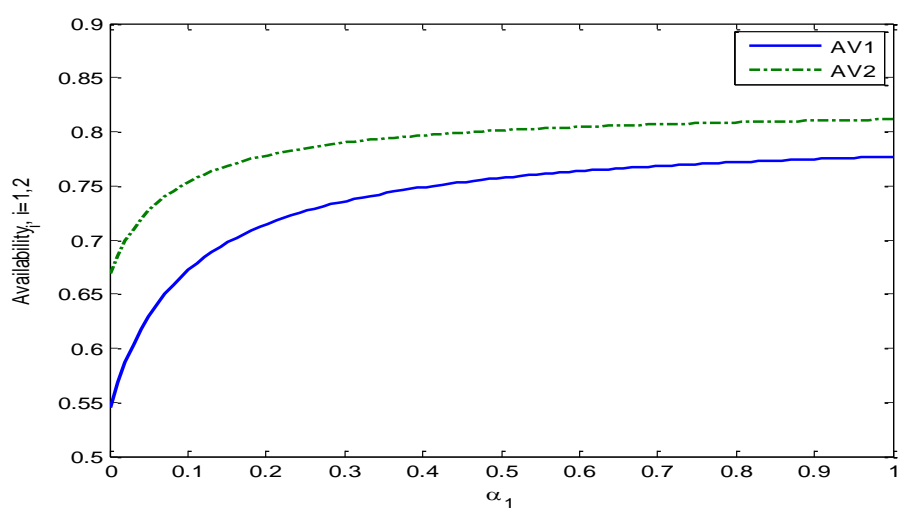

Fig. 6: Availability $_{i}$ versus repair rate $\alpha_{1}$ 


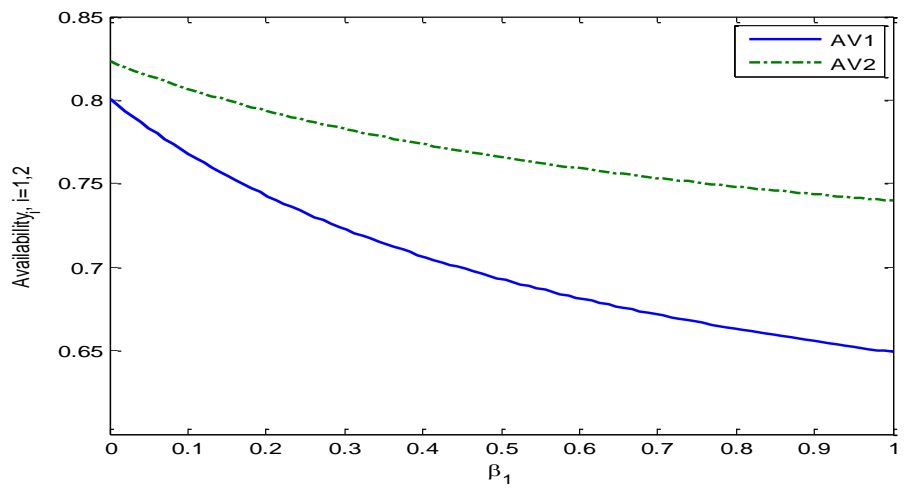

Fig. 7: Availability $_{i}$ versus failure rate $\beta_{1}$

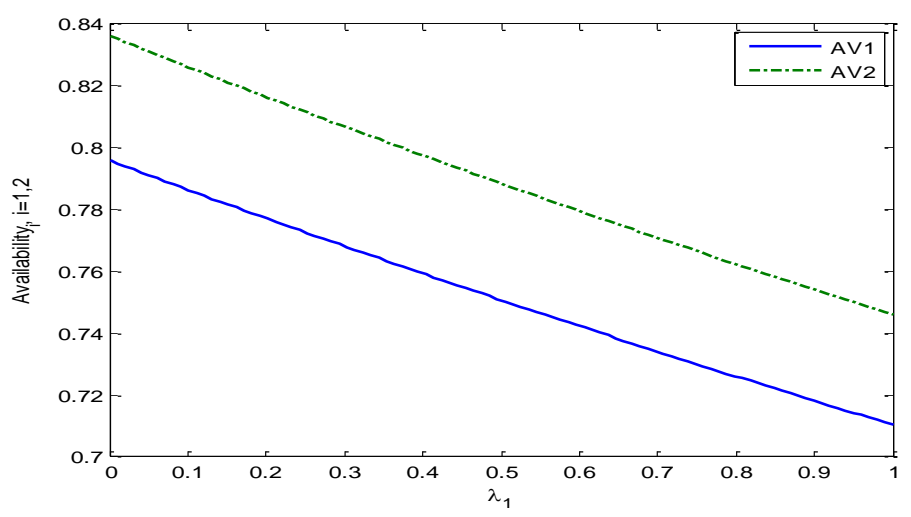

Fig. 8: A vailability $_{i}$ versus common cause failure $\lambda_{1}$

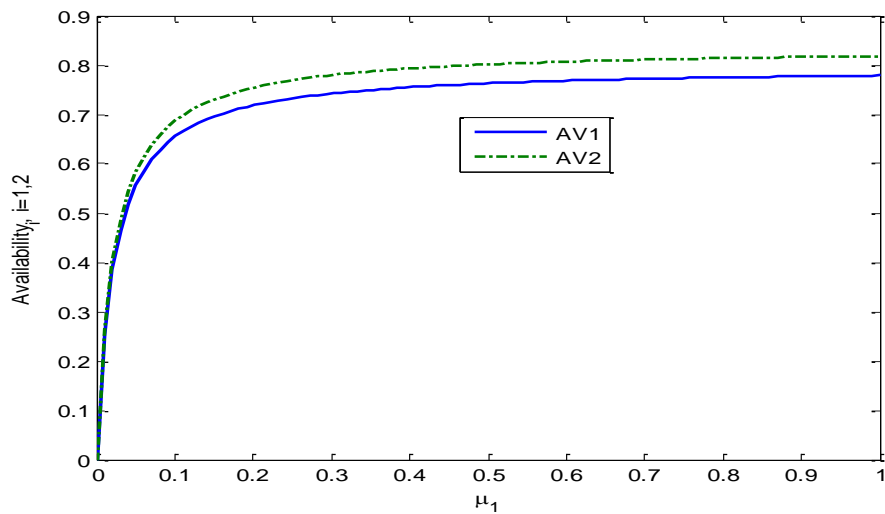

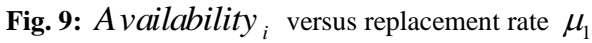

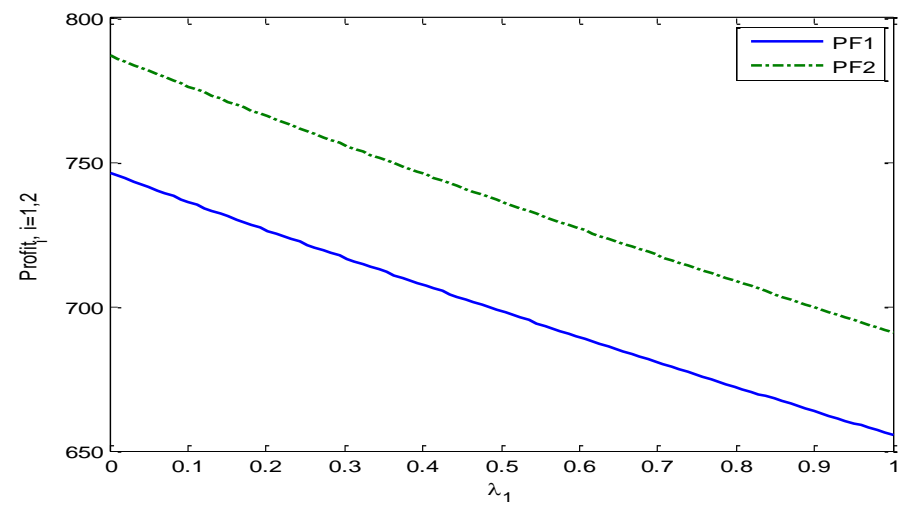

Fig. 10: Profit ${ }_{i}$ versus common cause failure rate $\lambda_{1}$ 


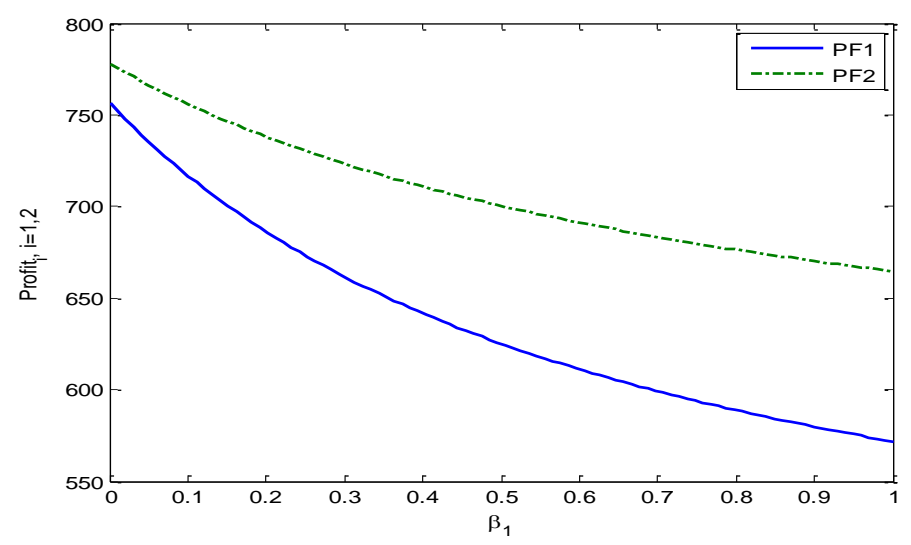

Fig. 11: Profit ${ }_{i}$ versus failure rate $\beta_{1}$

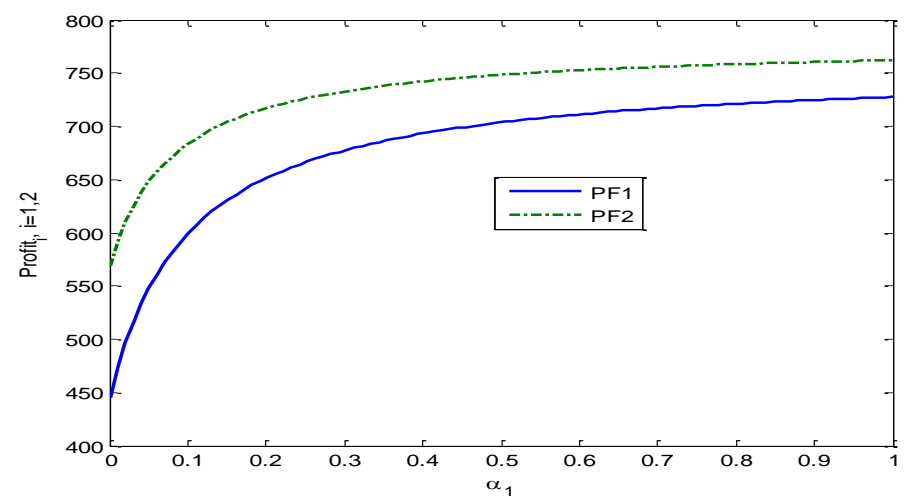

Fig. 12: Profit $_{i}$ versus repair rate $\alpha_{1}$

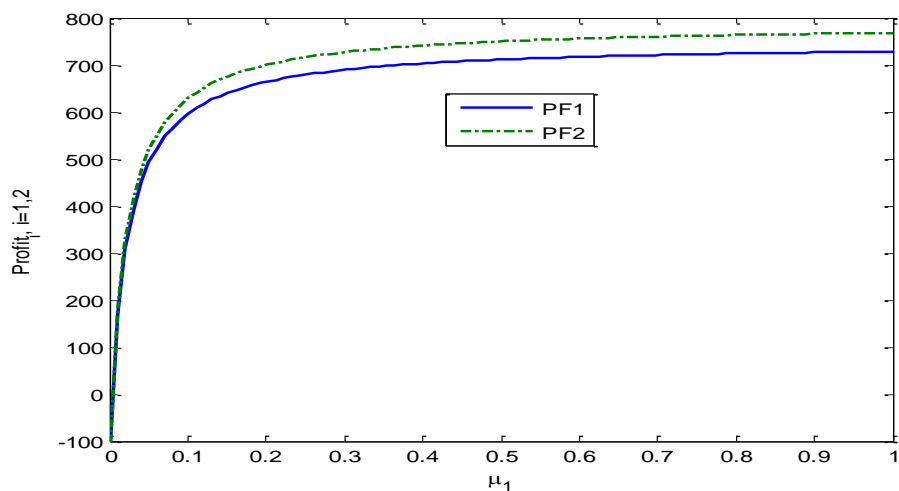

Fig. 13: Profit ${ }_{i}$ versus replacement rate $\mu_{1}$

Numerical results of $M T S F_{J}$ for configuration I and II are shown in Figures 3 - 5.Figures $3-4$ show that the $M T S F_{J}$ for configuration I and II decreases as $\lambda_{1}$ and $\beta_{1}$ increases. On the other hand, Fig. 5 show that the MTSF $F_{J}$ increases as $\alpha_{1}$ increases. It is evident from Figures $3-5$ that the optimal configuration using MTSF $F_{J}$ value is configuration II. Results of the $A_{V J}(\infty)$ for configuration I and II are shown in Figures 6, 7, 8 and 9 respectively. Figures 6 and 9 show that $A_{V J}(\infty)$ increases as $\alpha_{1}$ and $\mu_{1}$ increases for both configurations. On the other hand, Figures 7 and 8 show that $A_{V J}(\infty)$ decreases as $\beta_{1}$ and $\lambda_{1}$ increases for both configurations. Here the optimal configuration with respect to $A_{V J}(\infty)$ is configuration II. Graphical study of the $P F_{J}$ for configuration I and II are shown in Figures10, 11, 12 and 13 respectively. Figures 12 and 13 show that $P F_{J}$ increases as $\alpha_{1}$ and $\mu_{1}$ increases for both configurations. On the other hand, Figures 10 and 11 show that $P F_{J}$ decreases as $\lambda_{1}$ and $\beta_{1}$ increases for both configurations. Here the optimal configuration with respect to $A_{V J}(\infty)$ is configuration II. 
We can see from graphical study of system behavior that configuration II is the optimal configuration for 2-out-of-3 system in this study.

\section{Conclusion}

In this paper, we constructed two dissimilar cold standby systems configurations to study the effectiveness of each model.

Configuration I is 3-out-of-4 cold standby system while configuration II is 3-out-of-5 cold standby system. Explicit Expressions MTSF, steady-state availability, busy period and profit function for the two configurations were derived and comparative analysis was also performed numerically. It is evident from Figures $3-13$ that the optimal configuration is.

Configuration II using $\operatorname{MTSF}_{J}, A_{V J}(\infty)$ and $P F_{J}$.

\section{References}

[1] A.Khatab, N. Nahas, M.Nourelfath, Availbilty of k-out-of-n: G systems with non-identical components subject to repair priorities.Reliab. Eng. Syst. Safety. 94, (2009) 142-151.

[2] B.S. Dhillon, O.C. Anude, Common cause failure analysis of a non-identical two unit parallel system with arbitrary distributed times. Microelectronics and reliability, Vol. 33, No. 1, (1993) 87-103.

[3] D.S.Vashisth, Analysis of redundant system with common cause failures. International Journal of engineering Science and Technology, Vol. 3, No. 12, (2011) 8247-8254.

[4] G.R. Jahanshahloo,M.H.Behzad, M. Mirbolouki,M. Ranking stochastic efficient DMU based on reliability, International Journal of Industrial Mathematics, Vol.2,No.4,(2010) 263-270.

[5] G.S. Mokaddis, M.S. El Sherbeny, E.Al-Esayey,E. Compare between two unit cold standby and warm standbyb outdoor electric power systems in changing weather, Journal of Mathematics and Statistics, 6(1), (2010) 17-22.

[6] I.Yusuf, Comparison of some reliability characteristics between redundant systems requiring supporting units for their operation. Journal of Mathematical and Computational Sciences, 3(1), (2013) 216-232.

[7] I.Yusuf, B.Yusuf, Evaluation of reliability characteristics of two dissimilar network flow systems. Applied Mathematical Sciences, 7(40), (2013) 1983-1999 [7].

[8] J.C.Ke, and Y.K.Chu, Comparative analysis of availability for a redundant repairable system. Applied Mathematics and Computation, 188, (2007) 332-338.

[9] J.Shao, L.R. Lamberson, (Modeling shared-load k-out-of-n: G systems. IEEE Trans. Reliab., 40, (1991) : DOI:10.1109/24.87129.

[10] K.H.Wang, C.H Hseih, C.H. Liou, Cost benefit analysis of series systems with cold standby components and a repairable service station, Quality technology and quantitative management, 3(1), (2006) 77-92.

[11] K.H.Wang, Y.J.Chen, Comparative analysis of availability between three systems with general repair times, reboot delay and switching failures. Applied Mathematics and Computation, 215, (2009) 384-394.

[12] K.H.Wang, T.C, Yen,Y.C.Fang, Comparison of Availability between two systems with warm standby units and different imperfect coverage, Quality technology and quantitative management, 9(3), (2012) 265-282.

[13] K.M. El-Said, Cost analysis of a system with preventive maintenance by using Kolmogorov's forward equations method. American Journal of Applied Sciences, Vol.5, No.4, (2008) 405-410.

[14] M.A. Abdo, M. Hori, Reliability of Using 3-D Woven Polypropylene Fiber in Strengthening of a Large-Scale RC Structure Subjected to Seismic Loads, Arabian Journal for Science and Engineering Volume 37, Issue 3, (2012) 505-520 DOI10.1007/s13369-012-0208-3.

[15] M.Y.Haggag, Cost analysis of a system involving common cause failures and preventive maintenance. Journal of Mathematics and Statistics, Vol. 5, No.4, (2009) 305-310.

[16] Y.,Barron, E. Frostig, B. Levikson, Analysis of $\mathrm{r}$ out of $\mathrm{n}$ systems with several repairmen, exponential life times and phase type repair times: an algorithmic approach. Eur. J. Oper. Res. Algorithm Approach 169, (2006) 202-225.

[17] Z.Iravani, A generalized model in the performing evaluation of decision making sub-units, International Journal of Industrial Mathematics, Vol.2, No.3, (2010) 189-198. 